\title{
Fluctuating and intermittent facial weakness following a local anaesthetic
}

\author{
M. KINSBOURNE AND GEOFFREY RUSHWORTH \\ From Mount Vernon Hospital, Northwood, Middlesex, and \\ from the Neurological Research Unit, Churchill Hospital, Oxford
}

Considering the extensive use of local anaesthetics, especially in dental anaesthesia, it is surprising that injury to peripheral nerves, as a result of the injection, appears to be rare. During local anaesthesia of the maxillary nerve territory it is not uncommon for there to be a little weakness of the cheek and lip muscles, presumably due to spread of local anaesthesia into the proximity of facial nerve branches, but this invariably recovers as the effect of the local anaesthetic wears off.

Muscle weakness, which is the result of a partial lesion of a peripheral nerve, is usually fairly constant and is relatively unaffected by use of the muscle, though the muscle may fatigue more readily. Cooling of the muscle, however, may increase its weakness a little.

The patient we are about to describe appears to show two unique features. First, a partial lesion of the branches of the facial nerve was sustained during an injection for dental anaesthesia. Secondly, the weakness of the affected muscles was considerably increased by activity of facial muscles or by cooling the cheek.

J.M., a 32-year-old married secretary, during a course of dental treatment a year ago, was given an injection of local anaesthetic in the area of the right upper jaw for a filling of a right upper molar. This did not result in the expected numbness but, instead, was followed by local swelling, with mild tenderness, lasting for several days. Since then the patient has been subject to a fluctuating weakness of facial muscles in relation to the right corner of the mouth. This takes the form of a variable drooping of the right corner of the mouth, and, during a severe episode, it is associated with a feeling of slackness in the right cheek and a tendency to bite it accidentally. When eating, food tends to collect in the hollow of the cheek. Speaking may be a little indistinct, due to inability to manipulate the lips correctly. The severe weakness does not usually last for more than a few minutes. There is no weakness elsewhere, no pain, no sensory change and, now, no local swelling.

Sometimes the local weakness may be marked and obvious. On other occasions it may be quire undetectable. Often the patient wakes up with the facial weakness, which may resolve in a matter of a few minutes. The weakness tends to recur many times each day. It is precipitated or aggravated by exposure of the cheek to cold. It is relieved by local warmth. Continuous speaking or brushing the teeth may also precipitate paresis. In the course of a year, the fluctuating paresis has not changed, either in overall frequency or in maximum severity. No relieving factors, other than local warmth and rest, have been found.

The patient has not suffered from any relevant previous diseases. In childhood she had an attack of poliomyelitis, leaving a residual weakness of the back muscles, with scoliosis convex to the left.

Examination showed J. M. to be a healthy-looking woman, without abnormalities other than the previously mentioned scoliosis and a slight drooping of the right corner of the mouth which became more obvious when she spoke. On detailed testing, weakness could be demonstrated only in the levators of the right corner of the mouth and the right buccinator. There was no cutaneous sensory change, and no abnormality of other cranial nerves. However, a point of slight tenderness could be located by pressure just below the right zygoma and anterior to the masseter muscle.

Radiographs of the facial bones showed no abnormality.

An intravenous injection of $10 \mathrm{mg}$. of Tensilon produced no detectable change in the muscle weakness. However, local heating of the right cheek, by means of a lamp, caused resolution of the weakness within two minutes; and local cooling, by means of an icebag, resulted in an equally rapid restoration of maximum weakness. Counting aloud to 50 also caused a distinct drooping of the right upper lip.

No other specific relieving measure has been found, and no operative exploration of the facial nerve has been undertaken.

Electromyograms were first recorded in March 1965, about one year after the onset of symptoms. The patient was seen on a cold day, after a long car journey, and a distinct lower facial paresis was apparent. No attempt to warm the face was made at that time.

The recordings were taken by means of two coaxial needle electrodes within the right levator angulae oris and the adjacent part of the right orbicularis oris. There was no spontaneous activity at rest. Maximum voluntary effort evoked only single units up to 300 microvolts in amplitude; they were very polyphasic and their mean duration (calculated for 20 potentials recorded from 


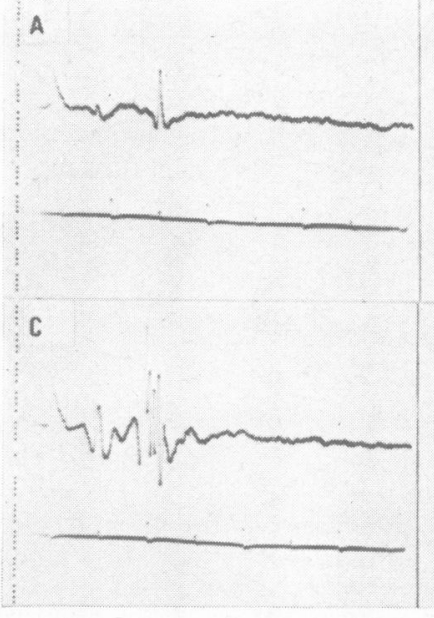

FIG. 1
B

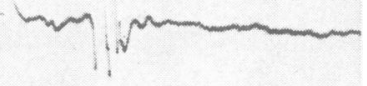

D

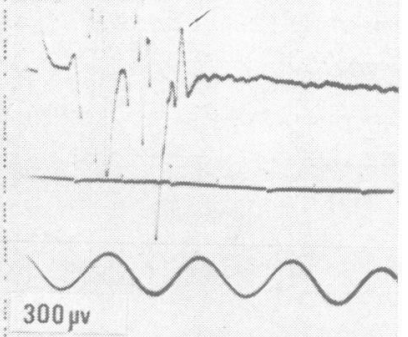

5 and 10 m.secs
FIG. 1. Electromyographic recordings from a coaxial needle electrode within the right levator angulae oris. The facial nerve was stimulated percutaneously near the angle of the jaw (conduction distance $9 \mathrm{~cm}$ ).

Stimulus strength progressively increased from $A$ to $D$. $A$ and $B$ show the low threshold late response (latency 10 msec.). $C$ and $D$ show also the higher threshold early response (latency $3.5 \mathrm{msec}$.).

FIG. 2. Electromyographic recordings from a coaxial needle electrode in the right levator angulae oris. The facial nerve was stimulated maximally, near the angle of the jaw. $(A)$ Before cooling of cheek.

(B) 1 min. after cooling. (C) 3 min. (D) 6 min. (E) 9 min. (F) Immediately after the withdrawal of cooling.

(G) 3 min. later. $(H) 6$ min. later.

Progressive fall in amplitude of all potentials occurred during cooling, but particularly of the early, high threshold component.

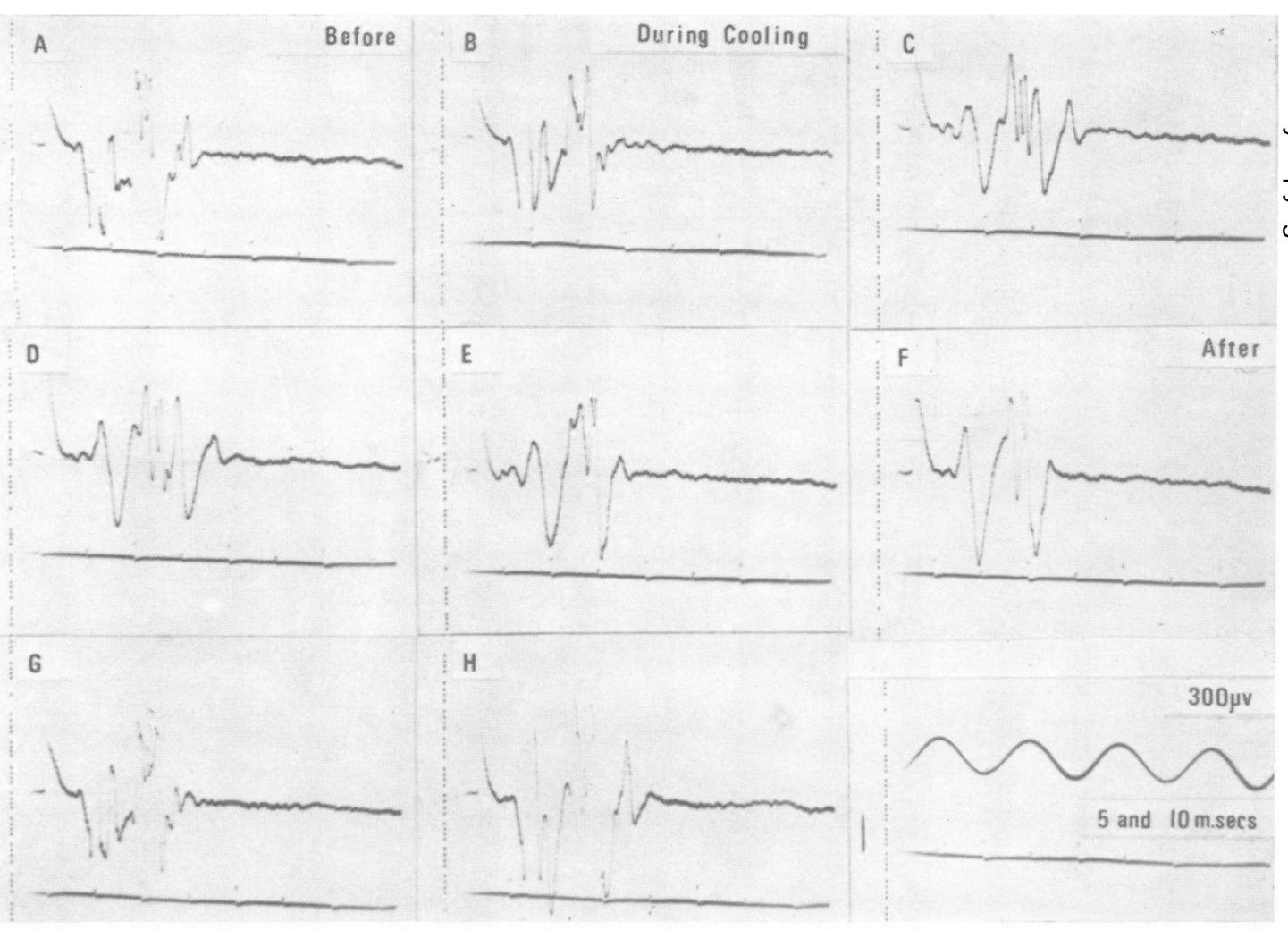




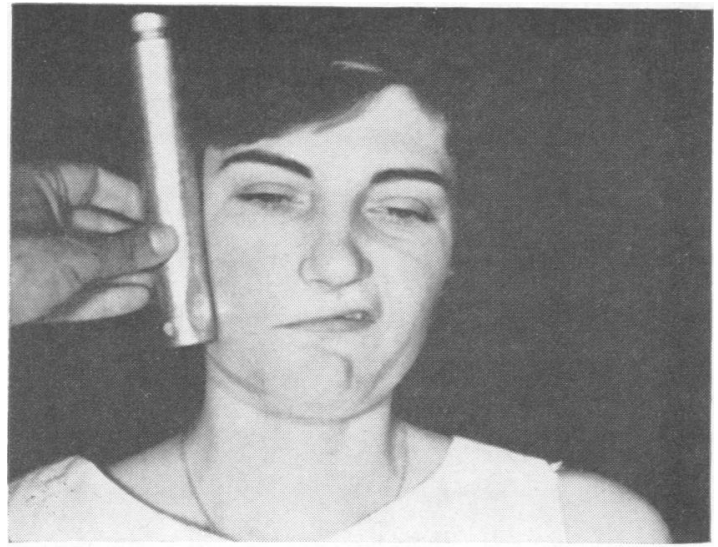

FIG. $3 \mathrm{a}$

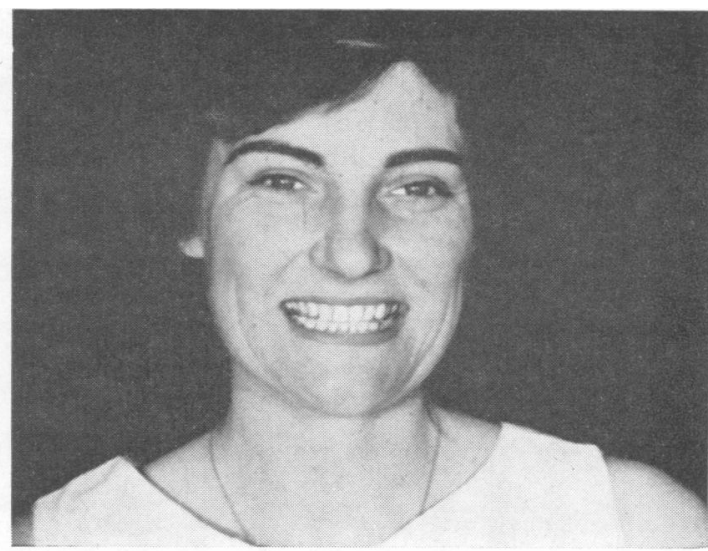

FIG. $3 b$ ice had been continued for almost 10 min. The patient is talking. Fig. 36 . Two min. later after removal of the tube. Complete recovery occurred under the hot lights required for colour filming.

several sites within the muscle) was $10 \mathrm{msec}$. (significantly greater than the normal mean of 6 msec. at this age).

Stimulation of the right facial nerve was performed percutaneously near the angle of the jaw some $9 \mathrm{~cm}$. away from the two recording electrodes. This evoked a very asynchronous response from the levator angulae oris, which was clearly divisible into two components. First, an early large component with a latency of 3.5 msec. (normal) was recorded, and then a second component with a latency of 8 to $10 \mathrm{msec}$. By reducing the stimulus strength it was possible to separate the second component in isolation at its prolonged latency. Surprisingly, the long latency response had the lower threshold to stimulation. (Fig. 1), and was recordable from several sites within this muscle and within the adjacent orbicularis oris.

The electromyographic findings showed that the muscles around the right side of the mouth were partly denervated, and the polyphasic long duration action potentials suggested that a little regeneration by sprouting had occurred.

By stimulation of the facial nerve proximally it was shown that the lowest threshold motor fibres evoked a response in the muscles with a prolonged latency, being delayed some 4-7 msec. longer than normal over a short length of nerve. This finding showed that there was a lesion of the facial nerve branches of such a type as to delay nerve impulses in some of the largest motor nerve fibres.

The patient was seen again in May 1965. On this occasion her face was first warmed by means of an infrared lamp. Recordings were taken once more from the right levator angulae oris. The right facial nerve was stimulated near the angle of the jaw and again at a low intensity of stimulation. It evoked a long latency response (Fig. 2), as on the previous occasion and, on increasing the stimulus strength, an early latency response eventually appeared at a latency of about $3.5 \mathrm{msec}$. Stimulus strength was then increased until a large early and a large late component were clearly visible in the recording. The right cheek was then cooled by means of a metal tube filled with ice. In the course of the next five minutes there was a considerable reduction of the amplitude of both the early and the late components, and the response became even more asynchronous and of longer duration than before cooling. In addition, the latency of the first response was prolonged to about $7 \mathrm{msec}$. On re-warming the face, the two responses rapidly increased in size and decreased to their previous latency.

The effects of cooling the cheek were also recorded by means of a $16 \mathrm{~mm}$. colour film (Fig. 3). Initially, with the hot lights required for this type of photography, little facial weakness was detectable clinically, but within about 10 minutes of cooling the cheek there was a dense right lower facial weakness.

\section{DISCUSSION}

Electromyography revealed clear-cut evidence of a lesion of the branch of the facial nerve destined for the levator angulae oris. It seems likely that the site of the lesion coincided with the tender spot in the cheek which had been there since the injection of local anaesthetic. A nerve lesion could, presumably, result from direct penetration of the nerve with the hypodermic needle and injection into it of a fairly large volume of solution. If this interpretation is correct, then the tender spot in the cheek was probably a small neuroma, resulting from total destruction of some nerve fibres, and compressing surviving nerve fibres. Such compression could result in delayed conduction along large diameter low threshold motor nerve fibres, as was demonstrated, and the ease with which increased nerve block could be induced by local cooling. 
The mechanism of fluctuating weakness is likely to be related to local vascular fluctuations in the region of impaired nerve function.

The stream of nerve impulses necessary to activate the lips during talking might push the oxygen requirements of the nerve to exceed the blood supply of this damaged and compressed region. However, under normal circumstances, large nerve fibres are very resistant to anoxia, taking 20-30 minutes to block.

Other possible mechanisms might include the shunting of blood away from the nerve to adjacent active muscles, or the neuroma region might be stretched by the actively contracting muscles, thus further obstructing the nerve's blood supply.
SUMMARY

A patient is described who developed a fluctuating weakness of the muscles of the right lip and cheek after a local anaesthetic injection for a minor dental procedure.

The muscle weakness was greatly intensified by local cooling and by talking.

Electromyographic and facial nerve conduction changes are reported.

The possible causation of this localized nerve lesion is discussed and the mechanism of the fluctuations.

We are very grateful to Mr. G. L. Fordyce for advice and for referring this patient to us for investigation. We also thank Professor Ritchie Russell for his helpful discussion of the case. 